\title{
Claudin 1 in Breast Tumorigenesis: Revelation of a Possible Novel “Claudin High" Subset of Breast Cancers
}

\author{
Yvonne Myal, ${ }^{1,2}$ Etienne Leygue, ${ }^{3}$ and Anne A. Blanchard ${ }^{1,2}$ \\ ${ }^{1}$ Department of Pathology, University of Manitoba, 770 Bannatyne Avenue, Winnipeg, Manitoba, Canada R3E 0W3 \\ ${ }^{2}$ Department of Physiology, University of Manitoba, 770 Bannatyne Avenue, Winnipeg, Manitoba, Canada R3E 0W3 \\ ${ }^{3}$ Department of Biochemistry and Medical Genetics, University of Manitoba, 770 Bannatyne Avenue, Winnipeg, \\ Manitoba, Canada R3E OW3 \\ Correspondence should be addressed to Yvonne Myal, myal@cc.umanitoba.ca
}

Received 22 September 2009; Revised 5 February 2010; Accepted 6 February 2010

Academic Editor: Amanda McCann

Copyright ( $) 2010$ Yvonne Myal et al. This is an open access article distributed under the Creative Commons Attribution License, which permits unrestricted use, distribution, and reproduction in any medium, provided the original work is properly cited.

Claudins are the major component of the tight junctions in epithelial cells and as such play a key role in the polarized location of ion channels, receptors, and enzymes to the different membrane domains. In that regard, claudins are necessary for the harmonious development of a functional epithelium. Moreover, defective tight junctions have been associated with the development of neoplastic phenotype in epithelial cells. Breakdown of cell-cell interactions and deregulation of the expression of junctional proteins are therefore believed to be key steps in invasion and metastasis. Several studies suggest that the claudins are major participants in breast tumorigenesis. In this paper, we discuss recent advances in our understanding of the potential role of claudin 1 in breast cancer. We also discuss the significance of a subset of estrogen receptor negative breast cancers which express "high" levels of the claudin 1 protein. We propose that claudin 1 functions both as a tumor suppressor as well as a tumor enhancer/facilitator in breast cancer.

\section{Breast Cancer}

Breast cancer remains one of the most commonly diagnosed cancers among women in North America [1]. Based on molecular, epidemiological, and histological observations, a morphological progression model for breast cancer has been assembled within the last decade [2-4]. This proposed model (Figure 1) outlines a continuum of lesions describing a stepwise progression of breast cancer, from epithelial hyperplasia, through atypical hyperplasia and ductal carcinoma in situ, to invasive carcinoma and eventually metastatic disease [24]. Despite many advances in the diagnosis and treatment of breast cancer, metastasis remains an insurmountable challenge. About $40 \%$ of women currently fail primary management strategies for early breast cancer and ultimately succumb to the disease.

The complex nature of the disease presentation and the limitations in identifying clinically relevant subsets of patients create major difficulties for current breast cancer diagnostic and therapeutic strategies. A growing understanding of the heterogeneous nature of this disease has stemmed primarily from cDNA microarray and immunohistochemical studies, which have led to a redefinition of breast cancer subsets [5-10]. To date, 5 distinct breast cancer categories have been identified (Table 1, [5-10]) based on ER/PR status, Her2, CK5/6, and EGFR expression. Therapeutically, these subtypes have been shown to display a wide variety of responses to different treatments $[6,11,12]$. The luminal A subtype (Table 1 ), which is more sensitive to hormones, has the most favorable outcome whereas the Her2 and the basal subtypes, which are not sensitive to hormones, are more aggressive, demonstrate the worst prognosis, and have fewer therapeutic options [13-15]. Evidently, the further identification of different subtypes of breast cancer will provide more therapeutic opportunities to match the characteristics of individual breast cancer patients, enhancing our ability to begin to offer individualized treatment to patients.

Two hypothetical models have been proposed to explain the evolution of breast cancer subtypes [18]. In the first model, the linear model, the cell of origin is the same for different tumor subtypes (and thus, tumor subtype is determined by acquired genetic and epigenetic events). In 


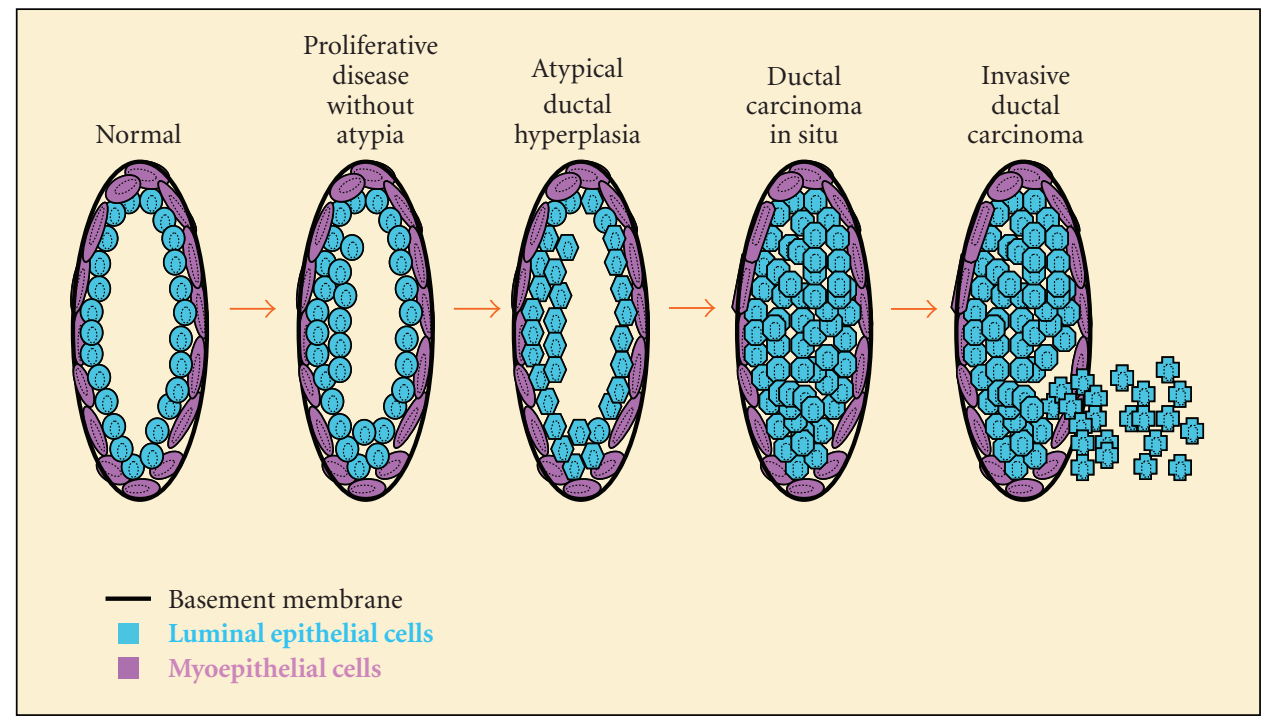

FIGURE 1: Hypothetical model of breast tumorigenesis and progression. The stepwise progression of breast cancer is described as a continuum of lesions from epithelial hyperplasia to invasive carcinoma and eventually metastasis.

TABLE 1: The five distinct subtypes of breast cancer and their corresponding phenotypes.

\begin{tabular}{|c|c|c|c|}
\hline Subtypes & Phenotype & BC Cell Lines & References \\
\hline Luminal A & ER+ve and/or PR+ve, Her2-ve & MCF7, T47D, ZR75-1 & {$[3,6,9,16]$} \\
\hline Luminal B & ER+ve and/or PR+ve, Her2+ve & MDA361, BT474, ZR75-30 & {$[3,6,9,16]$} \\
\hline Her2 overexpressing & ER-ve, PR-ve, Her2+ve & $\begin{array}{l}\text { HCC202, HCC1419, } \\
\text { HCC1569, HCC1954 }\end{array}$ & {$[3,6,16]$} \\
\hline Basal-like & $\begin{array}{l}\text { ER-ve, PR-ve, Her2-ve, CK5,6+ve } \\
\text { and/or EGFR+ve }\end{array}$ & BT20, MDA231, MDA468 & {$[3,6-8,16,17]$} \\
\hline Normal-like & $\begin{array}{l}\text { not clearly defined, similar to normal } \\
\text { epithelia, displays putative-initiating stem } \\
\text { cell phenotype }\end{array}$ & N/A & {$[3,6,10]$} \\
\hline
\end{tabular}

BC: breast cancer; ER: estrogen receptor; PR: progesterone receptor; Her2: human epidermal growth factor receptor 2; CK5,6: cytokeratin 5, 6; EGFR: epidermal growth factor receptor; +ve: positive; -ve: negative; N/A: not available.

the second model, the nonlinear model, the cell of origin is different (presumably a stem cell or a progenitor cell) for the different subtypes. Recent work by Jeselsohn et al. [19] has provided evidence to suggest that there are two populations of progenitor cells, one giving rise to the luminal-like breast cancers and the other to the basal-like breast cancers.

\section{Epithelial Mesenchymal Transition (EMT)}

The acquisition of the invasive phenotype is thought to mark the most significant change in breast cancer biology as it represents the first step towards the development of metastatic disease. As cells convert from the noninvasive to the invasive phenotype, they become anchorage independent and exhibit enhanced motility as well as increased aggressiveness, a process referred to as epithelial-mesenchymal transition (EMT).

During this transition, epithelial cells acquire a mesenchymal-like phenotype via disruption of intercellular adhesion and enhanced motility (for review see [20]). It is believed that cells switch from a keratin (epithelial) rich network to a vimentin (mesenchymal) rich network to facilitate their motility [20,21]. As for mesenchymal cells, in contrast to epithelial cells, they can individually migrate, penetrate into surrounding tissues, and spread to distant sites [20,22, 23]. The breakdown of cell-cell interactions and the deregulated expression of the junctional proteins are therefore believed to be key steps in invasion and metastasis [24, 25].

\section{Tight Junctions}

Tight junctions are the most apical of intercellular junctions and appear as a network of continuous and anastomosing filaments on the protoplasmic face of the plasma membrane [27] (Figure 2). They contribute to the transepithelial barrier that controls the transport of ions and small molecules through the paracellular pathway, a property referred to as the "barrier" function $[28,29]$. Tight junctions are also crucial for the organization of epithelial cell polarity, 


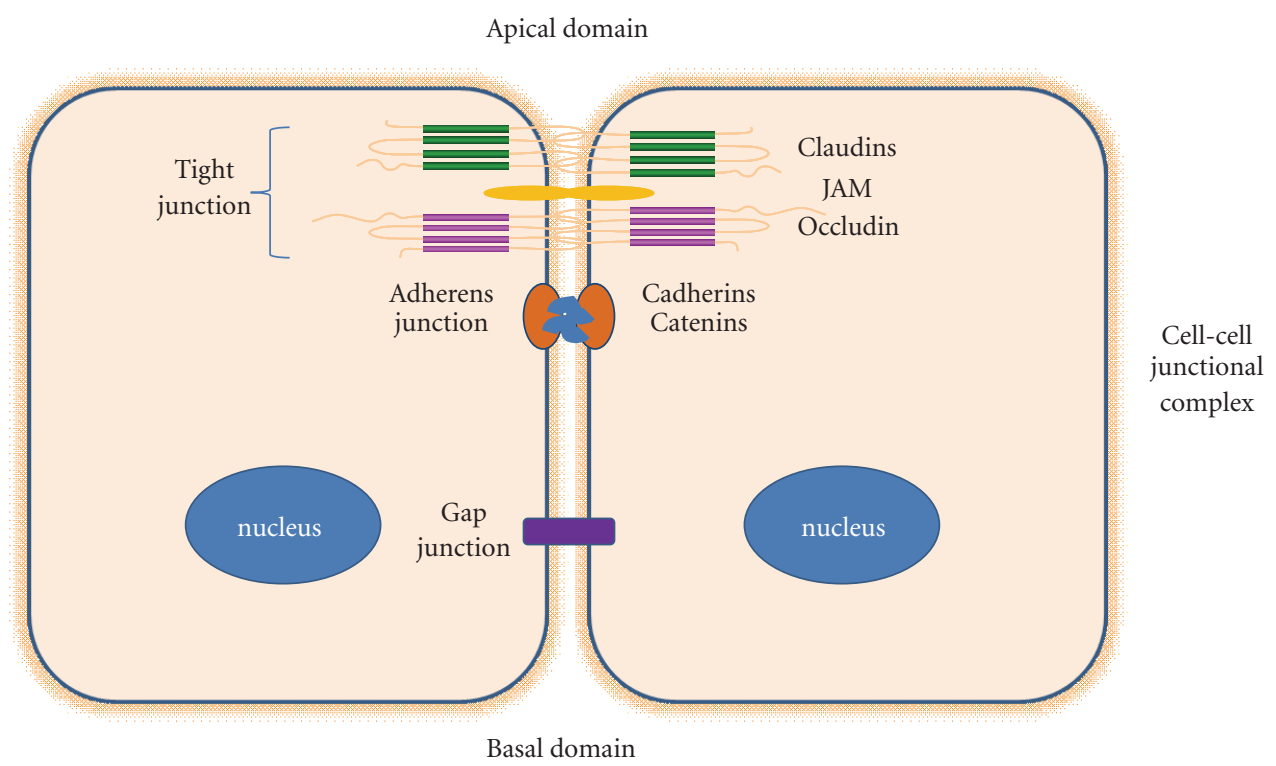

FIgure 2: Tight Junctions. Tight junctions are the most apical intercellular junctions in epithelial cells. The major molecular components of the tight junctions are the claudins, occludin, and the junctional adhesion molecule (JAM). Tight junction proteins in conjunction with adheren junction proteins (cadherins, catenins) form epithelial cell junctional complexes [26]. The gap junction is located basal to the adheren junction.

separating the plasma membrane into apical and basolateral domains [30-35]. As well, they are also critical for the polarized location of ion channels, receptors, and enzymes to the membrane domains necessary for structurally and functionally developed epithelia, a function referred to as the "fence" function $[28,36]$. Tight junctions are therefore essential for the tight sealing of cellular sheets and maintaining homeostasis [35]. Aside from maintaining cell polarity and paracellular functions, tight junction proteins are involved in recruiting signaling proteins [37]. Primarily, three types of integral membrane proteins constitute the tight junctions (Figure 2); the claudins, occludin and the junctional adhesion molecule(s), with the claudins and occludin being the two main molecular components in forming the tight junction strand. The junctional adhesion molecule is believed to function as the initial spatial cue for tight junction formation [32]. In conjunction with the adheren junction proteins, which are responsible for the mechanical adhesion between adjacent cells and the stabilization of the whole multicellular architecture, they constitute the apical junctional complex in epithelial tissue $[33,38]$.

\section{Tight Junction Proteins and Tumorigenesis}

A strong association between tight junction proteins and cancer development has been established. Alterations in the structure and function of tight junctions have indeed been reported in adenocarcinomas of various organs [39-41]. An absence of tight junctions or defective tight junctions has also been associated with the development of the neoplastic phenotype in epithelial cells [29, 33, 42]. Such observations are consistent with the accepted idea that the disruption of tight junctions leads to loss of cohesion, invasiveness, and the lack of differentiation, thereby promoting tumorigenesis. Currently, most knowledge regarding the role of junctional proteins in cancer, and more specifically breast cancer, has stemmed from studies on the major adheren junction protein, E cadherin (for review see $[20,33]$ ). The downregulation of $\mathrm{E}$ cadherin is believed to be an important molecular event during epithelial-mesenchymal transition [20]. In contrast to E cadherin, the role of the tight junction proteins is not well understood in breast cancer.

4.1. The Claudins. The claudins are the major component of the tight junction, and 24 members of this family of proteins have been identified to date $[26,27]$. They are small proteins ranging in size from 22 to $27 \mathrm{kD}$ and are encoded by at least 17 human genes (Table 2), located on 12 different chromosomes [43,44]. The distribution of the loci for the claudin genes among so many different chromosomes is interesting as generally many gene families have most, if not all of their members located on one chromosome $[45,46]$. The wide distribution may reflect the multifunctional characteristics of these proteins. Three claudin gene clusters are readily apparent on chromosome 3 (3q28), 4 (4q35.1), and 7 (7q11.23) and it is very likely that members within these clusters may have similar function and tissue specificity [47]. Aside from these three claudin gene clusters, there appears to be no other obvious pattern (Table 2). It is possible that the expansion of the claudin gene family in humans may have allowed for the acquisition of novel functions during evolution, as postulated for this gene family in teleost fish [48]. The claudins share a common transmembrane topology; each family member is predicted to possess four transmembrane domains with intracellular amino and carboxyl-termini in the cytoplasm 
TABle 2: Chromosomal locations of the 24 members of the claudin gene family and examples of their tissue-specific gene expression in normal and neoplastic tissues. Homologues for all members of this family (except CLDN21) are also found in chimpanzee, mouse, dog, rat, chicken, and fish.

\begin{tabular}{|c|c|c|c|c|c|c|c|c|c|}
\hline \multirow{3}{*}{ Gene } & \multirow{3}{*}{ Human Chromosomal Location } & \multicolumn{8}{|c|}{ Tissue Expression ${ }^{1}$} \\
\hline & & \multicolumn{2}{|c|}{ Breast } & \multicolumn{2}{|c|}{ Prostate } & \multicolumn{2}{|c|}{ Lung } & \multicolumn{2}{|c|}{ Liver } \\
\hline & & Normal & Cancer & Normal & Cancer & Normal & Cancer & Normal & Cancer \\
\hline CLDN1 & $3 q 28-q 29$ & 8.7 & 2.4 & 1.5 & 0.8 & 5.0 & 10.1 & 21.1 & 14.9 \\
\hline CLDN2 & $\mathrm{Xq} 22.3-\mathrm{q} 23$ & 0.5 & 1.1 & 0.7 & 1.2 & 1.3 & 2.5 & 0.0 & 0.0 \\
\hline CLDN3 & $7 q 11.23$ & 29.2 & 85.1 & 7.5 & 37.8 & 1.3 & 47.8 & 6.0 & 7.4 \\
\hline CLDN4 & $7 q 11.23$ & 134.5 & 192.8 & 6.0 & 27.2 & 10.0 & 70.4 & 0.0 & 14.9 \\
\hline CLDN5 & $22 \mathrm{q} 11.21$ & 8.7 & 3.2 & 0.7 & 0.4 & 7.5 & 2.5 & 0.0 & 0.0 \\
\hline CLDN6 & $16 \mathrm{p} 13.3$ & 0.3 & 0.5 & 0.0 & 0.0 & 0.0 & 0.0 & 0.0 & 0.0 \\
\hline CLDN7 & 17 p13 & 20.4 & 13.4 & 24.0 & 9.8 & 7.5 & 25.1 & 0.0 & 37.2 \\
\hline CLDN8 & $21 q 22.11$ & 0.9 & 0.4 & 0.7 & 0.4 & 0.0 & 0.0 & 0.0 & 0.0 \\
\hline CLDN9 & $16 \mathrm{p} 13.3$ & 0.3 & 1.7 & 0.0 & 0.0 & 0.0 & 0.0 & 0.0 & 0.0 \\
\hline CLDN10 & $13 q 31-q 34$ & 0.3 & 0.1 & 0.7 & 0.4 & 0.0 & 1.3 & 0.0 & 0.9 \\
\hline CLDN11 & $3 q 26.2-q 26.3$ & 1.7 & 1.0 & 1.5 & 0.8 & 0.0 & 0.0 & 0.0 & 2.8 \\
\hline CLDN12 & $7 q 21$ & 12.4 & 9.3 & 3.7 & 4.5 & 15.0 & 1.3 & 15.1 & 6.5 \\
\hline CLDN13* & N/A & N/A & N/A & N/A & N/A & N/A & N/A & N/A & N/A \\
\hline CLDN14 & $21 \mathrm{q} 22.3$ & 0.0 & 0.6 & 0.0 & 0.0 & 0.0 & 0.0 & 0.0 & 0.0 \\
\hline CLDN15 & $7 q 11.22$ & 1.9 & 2.5 & 0.7 & 2.8 & 0.0 & 7.5 & 0.0 & 1.9 \\
\hline CLDN16 & $3 q 28$ & 0.0 & 0.1 & 0.0 & 0.0 & 0.0 & 0.0 & 0.0 & 0.0 \\
\hline CLDN17 & $21 q 22.11$ & 0.2 & 0.1 & 0.0 & 0.0 & 0.0 & 0.0 & 0.0 & 0.0 \\
\hline CLDN18 & $3 q 22.3$ & 0.0 & 0.0 & 0.0 & 0.0 & 2.5 & 1.3 & 0.0 & 0.0 \\
\hline CLDN19 & $1 \mathrm{p} 34.2$ & 0.0 & 0.1 & 0.0 & 0.0 & 0.0 & 0.0 & 0.0 & 0.0 \\
\hline CLDN20 & $6 q 25$ & 0.2 & 0.7 & 0.0 & 3.3 & 0.0 & 1.3 & 0.0 & 1.9 \\
\hline CLDN21 & $4 q 35.1$ & N/A & N/A & N/A & N/A & N/A & N/A & N/A & N/A \\
\hline CLDN22 & $4 q 35.1$ & 2.6 & 5.5 & 7.5 & 6.5 & 3.8 & 5.0 & 9.0 & 6.5 \\
\hline CLDN23 & $8 \mathrm{p} 23.1$ & 2.1 & 5.7 & 14.2 & 23.2 & 11.3 & 8.8 & 3.0 & 11.2 \\
\hline CLDN24 & $4 q 35.1$ & N/A & N/A & N/A & N/A & N/A & N/A & N/A & N/A \\
\hline
\end{tabular}

${ }^{1}$ Relative gene expression (SAGE data) derived from the Cancer Genome Anatomy Project (CGAP [50]).

* Mouse only; N/A: not available; 0.0 : not detected

Numbers in columns indicate the number of tags/200,000 for each tissue/histology.

and two extracellular loops [33, 49]. The expression pattern of the claudin proteins is tissue specific [33]; however, most tissues express multiple claudins that can interact in either a homotypic or heterotypic fashion to form the tight junction strand [33, 50]. The exact combination of the claudin proteins within a given tissue determines the selectivity, strength and tightness of the tight junction $[33,51]$.

To date, only a few studies have addressed the role of claudins in breast cancer and findings on their function remain controversial $[47,52,53]$. In several cancers, including breast cancer, altered protein expression of some claudin family members has been demonstrated (for review see [33]). For example, protein expression of claudin 3 and 4 has been shown to be upregulated in invasive breast cancer [47] whereas, also in invasive breast cancer, the expression of the claudin 1 and 7 proteins were downregulated $[47,53,54]$.

4.2. Claudin 1. Knockout mice experiments have established that the tight junction protein claudin 1 , and not occludin, forms the backbone of the tight junction strand and is crucial for the epidermal barrier function $[35,55]$.
Expression of claudin 1 has been examined in a number of cancers (for review see [56]). Both an increase and a decrease in claudin 1 protein expression have been shown to be associated with tumorigenesis. In some cancers, including prostate [57], breast $[47,58]$, and melanocytic neoplasia [59], loss of claudin 1 has been associated with cancer progression and invasion, and the acquisition of the metastatic phenotype. In esophageal squamous cell carcinoma [60] decreased expression of claudin 1 correlated with recurrence and shorter disease free survival, whereas in lung cancer, claudin 1 was shown to suppress the expression of invasion/metastasis enhancers and increase expression of cancer invasion/metastasis suppressors, thereby providing supporting evidence to suggest that it functions as a cancer invasion/metastasis suppressor [61]. Conversely, in other cancers, such as papillary thyroid [62] oral squamous cell carcinoma [63], ovarian [64], colon [65, 66], melanoma [67], and gastric [68], overexpression of claudin 1 has been associated with aggressiveness and the increased malignant phenotype. Further, functional studies have shown that claudin 1 could recruit and promote the activation of the 
metalloproteinase MMP-2 $[63,69]$ and lead to a more aggressive phenotype in oral and ovarian cancer.

\section{Claudin 1 in Normal Breast and Breast Cancer}

In the normal mammary gland, tight junction proteins have mainly been investigated in relation to lactogenesis [32]. Previous work from our laboratory has identified claudin 1 as a highly upregulated gene during early mammary gland involution [70], and its expression was found to be tightly regulated during different stages of normal mouse gland development [71]. Recently, there has been an increased interest in the potential role of claudin 1 in breast cancer. Although studies are still relatively limited, there are a few critical reports which demonstrate a clear association between claudin 1 expression and breast cancer progression. The majority of studies point to a downregulation or complete loss of claudin 1 expression in malignant invasive human breast cancers $[47,53]$, and in some human breast cancer cell lines [72]. A correlation between claudin 1 down regulation and disease recurrence was also recently reported [73]. Additionally, functional studies also suggest that claudin 1 may be a key player in breast tumorigenesis. A down regulation of claudin 1 gene expression was shown to lead to neoplastic transformation of breast epithelial cells [74]. As well, the re-expression of claudin 1 alone was shown to be sufficient to induce apoptosis in a human breast cancer cell line [75]. It has also been suggested that claudin 1 alone might be sufficient to exert a tight junction-mediated gate function in metastatic tumor cells even in the absence of other tight junction-associated proteins [52]. In addition, subcellular localization of claudin 1 has been shown (by us [76] and others $[47,76,77])$ to be disrupted in invasive breast cancer leading to a detection of this protein in the cytoplasm. Interestingly, an association between claudin 1 and epithelial mesenchymal transition has recently been established. As with E cadherin $[78,79]$ the transcription factors, slug and snail, key markers of epithelial mesenchymal transition, were shown to bind to the claudin 1 promoter resulting in the repression of its activation [80].

Additional work from our laboratory has also provided evidence to show that claudin 1 expression in breast cancer is even more complex than originally thought. Using tissue microarray strategies, we showed that in a cohort of human invasive breast cancers exhibiting mixed pathological lesions (340 biopsies, the largest examined to date), only a small percentage of tumors express claudin 1 protein. The frequency of claudin 1 positive tumors was significantly lower than the frequency of tumors observed as positive for claudin 3 and 4, two family members previously shown to be overexpressed in invasive human breast cancer [47].

Since ER status is often considered an important classifier of breast cancers (Table 1), we wanted to determine whether there was any association between estrogen/estrogen receptor and claudin 1. We showed that in ER+ve breast cancers (189 biopsies), a significantly small number of tumors (5\%) were positive for claudin 1 expression, while in the ER-ve tumors (151 biopsies), the frequency of positive tumor staining for claudin 1 was significantly higher (39\%) [76]. A positive association was also found with EGFR, a marker of poor prognosis. Surprisingly, a significant correlation was also found with claudin 1 and markers of the basal-like subtype of breast cancers [76, 81], an aggressive subtype of breast cancer associated with the worst prognosis and reduced patient survival. We also demonstrated for the first time that in the estrogen receptor positive (ER+ve) human cell line, MCF7, that claudin 1 expression was down regulated by estrogen in vitro (unpublished data).

\section{Is Claudin 1 Much More than a Tumor Suppressor in Breast Tumorigenesis?}

Both an over and an underexpression of claudin 1 have been observed in different types of cancers $[57,59,62,63$, $65-67,69]$, outlining the complexity of its potential role in carcinogenesis. In breast cancer, the majority of studies published to date, though limited in numbers, show that partial or total loss of claudin 1 expression correlates with increased malignant potential and invasiveness and with recurrence of disease $[47,73]$. As well, the re-expression of claudin 1 in breast cancer cells was demonstrated to induce apoptosis [75]. Additionally, our tissue microarray studies showed that a significantly low frequency of human invasive breast cancers was positive for claudin 1 expression [76]. Altogether, these studies provide supporting evidence to suggest that claudin 1 functions as a tumor suppressor in breast tumorigenesis.

Paradoxically, our laboratory has also provided evidence to suggest that the role of claudin 1 in breast cancer may be much more than a tumor suppressor. We showed in our TMA studies that the frequency of claudin 1 positive tumors was significantly higher in ER-ve breast cancers than in ER+ve breast cancers [76]. To our knowledge these studies are the first report to address claudin 1 expression in breast cancer in the context of ER status. We further showed that claudin 1 positivity (as well as claudin 4) was significantly associated with the basal-like subtype of breast cancers, one of the most aggressive subtypes [76]. Of note, in a recent study by Kulka et al., 2008, it was demonstrated that claudin 4 expression was significantly higher in the basal-like subtype of breast cancers [81]. Since claudin 1 is generally considered to be a "tumor suppressor" in breast cancer, our observations were unexpected. How can such observations be rationalized? There are a few possible scenarios that may explain these findings. First, it is plausible that during tumorigenesis, not all tumor cells lose claudin 1 expression. In line with the proposed nonlinear model of breast cancer subtypes [18], it is possible that the cells which retain claudin 1 expression are the cells already predetermined to become ER-ve basal-like breast cancers. Then, in these cells, the role of claudin 1 may be that of a tumor promoter rather than a tumor suppressor. On the other hand, if one considers the linear model of breast cancer subtypes [18], tumor cells are believed to progress from ER+ve to ER-ve as the cancer advances. Then, is the increased frequency of claudin 1 positive tumors in the ER-ve cohort attributed to a re-expression of claudin 1 in these tumors? Such a 


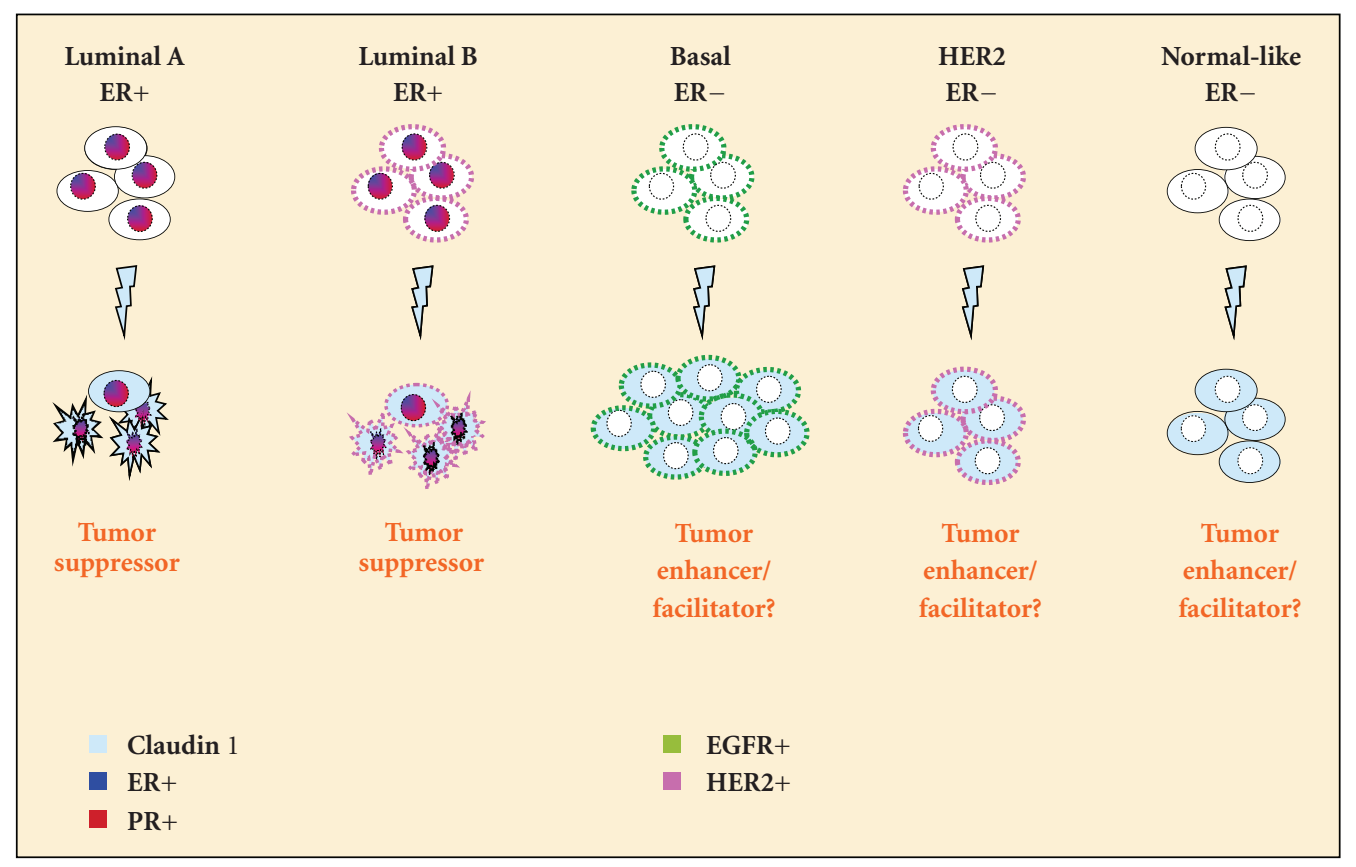

FIGURE 3: A hypothetical model of claudin 1 expression in different breast cancer subtypes. This model proposes that claudin 1 is a tumor suppressor in the ER+ve subtypes of breast cancer (luminal A and B) and a tumor enhancer/facilitator in the ER-ve subtypes (basal-like, Her2 over expressing, normal-like). Whereas a significant association between claudin 1 and the basal-like subtype has been demonstrated, this is yet to be determined for the Her2 over expressing and the normal-like subtypes.

concept is supported by the significantly higher expression of claudin 1 staining (an indicator of protein expression) that was observed in the ER-ve tumors. Here, the tumor suppressor function of the re-expressed claudin 1 is thus eliminated and now replaced by a tumor enhancing function, thereby facilitating breast tumorigenesis. This re-expression of claudin 1 could be attributed to a number of mitigating factors including genetic mutation in the claudin 1 gene or epigenetic modification of the protein. However, sequence analysis studies of the coding region of claudin 1 [53] in both sporadic and hereditary breast patients failed to identify any significant mutation that may be responsible for altering the claudin 1 gene expression in breast tumors. One interesting possibility is that the higher level of the claudin 1 protein could be due to a defective interacting partner resulting in the inability of claudin 1 to be transported back to the membrane where it may escape further down regulation by other factors, and therefore leading to an accumulation of claudin 1 in the cytoplasm. The latter has recently been demonstrated for E cadherin [82]. Furthermore, the exact combination of claudin proteins within a given tissue is thought to determine the strength of the tight junction [51]. Thus, one of the consequences of this aberrant accumulation of claudin 1 in the ER-ve invasive breast cancers may be a redefinition of the makeup of the tight junction, further undermining the integrity of the junction and thereby further facilitating tumor progression. Taken together, it appears that the role of claudin 1 extends beyond that of a tumor suppressor. We would like to propose that claudin 1 functions both as a tumor suppressor as well as a tumor enhancer/facilitator in breast cancer. We further propose that its tumor facilitating role is particularly associated with the ER-ve breast cancer subtypes (Figure 3).

Recently, a "claudin low" subtype of breast cancer has been identified that was primarily ER-ve and exhibited low expression of claudin 3, 4, and 7 [83]. We would further like to propose a "claudin high" subtype that is ER-ve and which exhibits a high expression of claudin 1 protein (and claudin 4). This subtype would include the basal-like breast cancers and exclude the ER+ve luminal subtypes. The clinical significance of a high expression of the claudin 1 protein in ER-ve breast cancers and its association with the basal-like subtype may identify its potential use as a diagnostic and prognostic indicator for a particular breast cancer subset.

\section{Future Perspectives}

Mounting evidence suggests that claudin 1 has a unique role in breast cancer and breast cancer progression. Since claudin 1 is a transmembrane protein with two large extracellular loops, it is a potentially attractive candidate for use in therapeutic strategies. As we begin to address the role of claudin 1 in breast cancer progression we are left with many unanswered questions. What is the expression level of the claudin 1 protein in different histological subtypes of breast cancer? What triggers its regulation and causes claudin 1 to switch its role at different stages of breast cancer progression? Why does claudin 1 accumulate in the cytoplasm? Is there a mutation in the claudin gene or protein in the basal-like subtype of breast cancer? Does claudin 1 work in concert with other claudins or junctional proteins such as E cadherin? Is there cross-talk with the estrogen 
receptor pathway? Clearly, more detailed functional analysis studies are warranted both in vitro and in vivo.

One can only predict that more breast cancer subtypes will be identified in the near future and a clearer understanding of the cellular and molecular changes occurring during breast tumorigenesis will be critical for facilitating more effective patient management and directly impacting on reducing mortality rates. Only through such understanding will new biomarkers be identified that will report on metastatic changes, breast cancer progression and serve as therapeutic targets ultimately leading to specific and individualized patient management.

\section{Acknowledgment}

The authors gratefully acknowledge funding for this work which was provided by the Manitoba Medical Services Foundation, the Paul Thorlakson Foundation, and the Canadian Breast Cancer Foundation.

\section{References}

[1] “Canadian Cancer Statistics," 2006, http://www.cancer.ca.

[2] D. C. Allred, Y. Wu, S. Mao, et al., "Ductal carcinoma in situ and the emergence of diversity during breast cancer evolution," Clinical Cancer Research, vol. 14, no. 2, pp. 370378, 2008.

[3] M. Hu, J. Yao, D. K. Carroll, et al., "Regulation of in situ to invasive breast carcinoma transition," Cancer Cell, vol. 13, no. 5, pp. 394-406, 2008.

[4] P. T. Simpson, J. S. Reis-Filho, T. Gale, and S. R. Lakhani, "Molecular evolution of breat cancer," Journal of Pathology, vol. 205, no. 2, pp. 248-254, 2005.

[5] G. Finak, S. Sadekova, F. Pepin, et al., "Gene expression signatures of morphologically normal breast tissue identify basal-like tumors," Breast Cancer Research, vol. 8, article R58, 2006.

[6] M. L. Kwan, L. H. Kushi, E. Weltzien, et al., "Epidemiology of breast cancer subtypes in two prospective cohort studies of breast cancer survivors," Breast Cancer Research, vol. 11, article R31, 2009.

[7] T. O. Nielsen, F. D. Hsu, K. Jensen, et al., "Immunohistochemical and clinical characterization of the basal-like subtype of invasive breast carcinoma," Clinical Cancer Research, vol. 10, no. 16, pp. 5367-5374, 2004.

[8] C. M. Perou, T. Sorlie, M. B. Eisen, et al., "Molecular portraits of human breast tumours," Nature, vol. 406, no. 6797, pp. 747$752,2000$.

[9] T. Sorlie, R. Tibshirani, J. Parker, et al., "Repeated observation of breast tumor subtypes in independent gene expression data sets," Proceedings of the National Academy of Sciences of USA, vol. 100, pp. 8418-8423, 2003.

[10] K.-D. Yu, Z.-Z. Shen, and Z.-M. Shao, "The immunohistochemically "eR-negative, PR-negative, HER2-negative, CK5/6negative, and HER1-negative" subgroup is not a surrogate for the normal-like subtype in breast cancer," Breast Cancer Research and Treatment, vol. 118, no. 3, pp. 661-663, 2009.

[11] A. H. Bild, J. S. Parker, A. M. Gustafson, et al., "An integration of complementary strategies for gene-expression analysis to reveal novel therapeutic opportunities for breast cancer," Breast Cancer Research, vol. 11, article R55, 2009.

[12] J. Hugh, J. Hanson, M. C. U. Cheang, et al., "Breast cancer subtypes and response to docetaxel in node-positive breast cancer: use of an immunohistochemical definition in the BCIRG 001 trial," Journal of Clinical Oncology, vol. 27, no. 8, pp. 1168-1176, 2009.

[13] R. I. Nicholson and S. R. Johnston, "Endocrine therapycurrent benefits and limitations," Breast Cancer Research and Treatment, vol. 93, supplement 1, pp. S3-S10, 2005.

[14] E. A. Rakha, S. E. Elsheikh, M. A. Aleskandarany, et al., "Triple-negative breast cancer: distinguishing between basal and nonbasal subtypes," Clinical Cancer Research, vol. 15, no. 7, pp. 2302-2310, 2009.

[15] T. Vargo-Gogola and J. M. Rosen, "Modelling breast cancer: one size does not fit all," Nature Reviews Cancer, vol. 7, no. 9, pp. 659-672, 2007.

[16] J. Kao, K. Salari, M. Bocanegra, et al., "Molecular profiling of breast cancer cell lines defines relevant tumor models and provides a resource for cancer gene discovery," PLoS ONE, vol. 4, no. 7, article e6146, 2009.

[17] E. A. Rakha and I. O. Ellis, "Triple-negative/basal-like breast cancer: review," Pathology, vol. 41, no. 1, pp. 40-47, 2009.

[18] K. Polyak, "Breast cancer: origins and evolution," Journal of Clinical Investigation, vol. 117, no. 11, pp. 3155-3163, 2007.

[19] R. Jeselsohn, N. E. Brown, L. Arendt, et al., "Cyclin D1 kinase activity is required for the self-renewal of mammary stem and progenitor cells that are targets of MMTV-ErbB2 tumorigenesis," Cancer Cell, vol. 17, no. 1, pp. 65-76, 2010.

[20] M. Guarino, B. Rubino, and G. Ballabio, "The role of epithelial-mesenchymal transition in cancer pathology," Pathology, vol. 39, no. 3, pp. 305-318, 2007.

[21] M. I. Kokkinos, R. Wafai, M. K. Wong, D. F. Newgreen, E. W. Thompson, and M. Waltham, "Vimentin and epithelial-mesenchymal transition in human breast cancerobservations in vitro and in vivo," Cells Tissues Organs, vol. 185, no. 1-3, pp. 191-203, 2007.

[22] B. Boyer, A. M. Valles, and N. Edme, "Induction and regulation of epithelial-mesenchymal transitions," Biochemical Pharmacology, vol. 60, no. 8, pp. 1091-1099, 2000.

[23] E. D. Hay, "An overview of epithelio-mesenchymal transformation," Acta Anatomica, vol. 154, no. 1, pp. 8-20, 1995.

[24] M. Mareel and A. Leroy, "Clinical, cellular, and molecular aspects of cancer invasion," Physiological Reviews, vol. 83, no. 2, pp. 337-376, 2003.

[25] J. M. Mullin, "Potential interplay between luminal growth factors and increased tight junction permeability in epithelial carcinogenesis," Journal of Experimental Zoology, vol. 279, no. 5, pp. 484-489, 1997.

[26] S. Tsukita, M. Furuse, and M. Itoh, "Multifunctional strands in tight junctions," Nature Reviews Molecular Cell Biology, vol. 2, no. 4, pp. 285-293, 2001.

[27] L. Gonzales-Mariscal, Tight Junctions, CRC Press, Boca Raton, Fla, USA, 2nd edition, 2001.

[28] J. M. Diamond, "Twenty-first Bowditch lecture. The epithelial junction: bridge, gate, and fence.," Physiologist, vol. 20, no. 1, pp. 10-18, 1977.

[29] H. Tobioka, H. Isomura, Y. Kokai, Y. Tokunaga, J. Yamaguchi, and N. Sawada, "Occludin expression decreases with the progression of human endometrial carcinoma," Human Pathology, vol. 35, no. 2, pp. 159-164, 2004.

[30] S. Eaton and K. Simons, "Apical, basal, and lateral cues for epithelial polarization," Cell, vol. 82, no. 1, pp. 5-8, 1995.

[31] B. Gumbiner, "Structure, biochemistry, and assembly of epithelial tight junctions," American Journal of Physiology, vol. 253, no. 6, pp. C749-C758, 1987.

[32] M. Itoh and M. J. Bissell, "The organization of tight junctions in epithelia: implications for mammary gland biology and 
breast tumorigenesis," Journal of Mammary Gland Biology and Neoplasia, vol. 8, pp. 449-462, 2003.

[33] P. J. Morin, "Claudin proteins in human cancer: promising new targets for diagnosis and therapy," Cancer Research, vol. 65, no. 21, pp. 9603-9606, 2005.

[34] K. Simons and S. D. Fuller, "Cell surface polarity in epithelia," Annual Review of Cell Biology, vol. 1, pp. 243-288, 1985.

[35] S. Tsukita and M. Furuse, "Claudin-based barrier in simple and stratified cellular sheets," Current Opinion in Cell Biology, vol. 14, no. 5, pp. 531-536, 2002.

[36] J. M. Andersen, M. S. Balda, and A. S. Fanning, "The structure and regulation of tight junctions," Current Opinion in Cell Biology, vol. 5, no. 5, pp. 772-778, 1993.

[37] L. L. Mitic and J. M. Anderson, "Molecular architecture of tight junctions," Annual Review of Physiology, vol. 60, pp. 121142, 1998.

[38] M. G. Farquhar and G. E. Palade, "Junctional complexes in various epithelia," The Journal of Cell Biology, vol. 17, pp. 375412, 1963.

[39] K. B. Hoover, S.-Y. Liao, and P. J. Bryant, "Loss of the tight junction MAGUK ZO-1 in breast cancer: relationship to glandular differentiation and loss of heterozygosity," American Journal of Pathology, vol. 153, no. 6, pp. 1767-1773, 1998.

[40] S. Polak-Charcon, J. Shoham, and Y. Ben-Shaul, "Tight junctions in epithelial cells of human fetal hindgut, normal colon, and colon adenocarcinoma," Journal of the National Cancer Institute, vol. 65, no. 1, pp. 53-62, 1980.

[41] A. P. Soler, R. D. Miller, K. V. Laughlin, N. Z. Carp, D. M. Klurfeld, and J. M. Mullin, "Increased tight junctional permeability is associated with the development of colon cancer," Carcinogenesis, vol. 20, no. 8, pp. 1425-1431, 1999.

[42] T. Kaihara, H. Kawamata, J. Imura, et al., "Redifferentiation and $\mathrm{ZO}-1$ reexpression in liver-metastasized colorectal cancer: possible association with epidermal growth factor receptorinduced tyrosine phosphorylation of ZO-1," Cancer Science, vol. 94, no. 2, pp. 166-172, 2003.

[43] National Center for Biotechnology Information, 2009, http:// www.ncbi.nlm.nih.gov/gene.

[44] European Bioinformatics Institute, 2009, http://www.ebi.ac .uk/embl/.

[45] J. O. Bishop, A. J. Clark, P. M. Clissold, S. Hainey, and U. Francke, "Two main groups of mouse major urinary protein genes, both largely located on chromosome 4," The EMBO Journal, vol. 1, no. 5, pp. 615-620, 1982.

[46] L. Hood, M. Steinmetz, and R. Goodenow, "Genes of the major histocompatibility complex," Cell, vol. 28 , no. 4, pp. 685-687, 1982.

[47] A. M. Tokes, J. Kulka, S. Paku, et al., "Claudin-1, -3 and 4 proteins and mRNA expression in benign and malignant breast lesions: a research study," Breast Cancer Research, vol. 7, no. 2, pp. R296-R305, 2005.

[48] Y. H. Loh, A. Christoffels, S. Brenner, W. Hunziker, and B. Venkatesh, "Extensive expansion of the claudin gene family in the teleost fish, Fugu rubripes," Genome Research, vol. 14, no. 7, pp. 1248-1257, 2004.

[49] S. Tsukita and M. Furuse, "Occludin and claudins in tightjunction strands: leading or supporting players?" Trends in Cell Biology, vol. 9, pp. 268-273, 1999.

[50] The Cancer Genome Anatomy Project, 2009, http://cgap.nci .nih.gov/.

[51] K. Morita, M. Furuse, K. Fujimoto, and S. Tsukita, "Claudin multigene family encoding four-transmembrane domain protein components of tight junction strands," Proceedings of the
National Academy of Sciences of the United States of America, vol. 96, no. 2, pp. 511-516, 1999.

[52] T. Hoevel, R. Macek, O. Mundigl, K. Swisshelm, and M. Kubbies, "Expression and targeting of the tight junction protein CLDN1 in CLDN1-negative human breast tumor cells," Journal of Cellular Physiology, vol. 191, no. 1, pp. 60-68, 2002.

[53] F. Kramer, K. White, M. Kubbies, K. Swisshelm, and B. H. F. Weber, "Genomic organization of claudin-1 and its assessment in hereditary and sporadic breast cancer," Human Genetics, vol. 107, no. 3, pp. 249-256, 2000.

[54] S. L. Kominsky, P. Argani, D. Korz, et al., "Loss of the tight junction protein claudin-7 correlates with histological grade in both ductal carcinoma in situ and invasive ductal carcinoma of the breast," Oncogene, vol. 22, no. 13, pp. 2021-2033, 2003.

[55] M. Furuse, M. Hata, K. Furuse, et al., "Claudin-based tight junctions are crucial for the mammalian epidermal barrier: a lesson from claudin-1-deficient mice," Journal of Cell Biology, vol. 156, no. 6, pp. 1099-1111, 2002.

[56] K. J. Hewitt, R. Agarwal, and P. J. Morin, "The claudin gene family: expression in normal and neoplastic tissues," BMC Cancer, vol. 6, article 186, 2006.

[57] G. M. Sheehan, B. V. S. Kallakury, C. E. Sheehan, H. A. G. Fisher, R. P. Kaufman Jr., and J. S. Ross, "Loss of claudins1 and -7 and expression of claudins- 3 and -4 correlate with prognostic variables in prostatic adenocarcinomas," Human Pathology, vol. 38, no. 4, pp. 564-569, 2007.

[58] K. Swisshelm, R. Macek, and M. Kubbies, "Role of claudins in tumorigenesis," Advanced Drug Delivery Reviews, vol. 57, no. 6, pp. 919-928, 2005.

[59] M. L. Cohn, V. N. Goncharuk, A. H. Diwan, P. S. Zhang, S. S. Shen, and V. G. Prieto, "Loss of claudin-1 expression in tumorassociated vessels correlates with acquisition of metastatic phenotype in melanocytic neoplasms," Journal of Cutaneous Pathology, vol. 32, no. 8, pp. 533-536, 2005.

[60] K. Miyamoto, T. Kusumi, F. Sato, et al., "Decreased expression of claudin-1 is correlated with recurrence status in esophageal squamous cell carcinoma," Biomedical Research, vol. 29, no. 2, pp. 71-76, 2008.

[61] Y.-C. Chao, S.-H. Pan, S.-C. Yang, et al., "Claudin-1 is a metastasis suppressor and correlates with clinical outcome in lung adenocarcinoma," American Journal of Respiratory and Critical Care Medicine, vol. 179, no. 2, pp. 123-133, 2009.

[62] J. Nemeth, Z. Nemeth, P. Tatrai, et al., "High expression of claudin-1 protein in papillary thyroid tumor and its regional lymph node metastasis," Pathology \& Oncology Research, vol. 16, no. 1, pp. 19-27, 2009.

[63] N. Oku, E. Sasabe, E. Ueta, T. Yamamoto, and T. Osaki, “Tight junction protein claudin-1 enhances the invasive activity of oral squamous cell carcinoma cells by promoting cleavage of laminin-5 $\gamma 2$ chain via matrix metalloproteinase (MMP)-2 and membrane-type MMP-1," Cancer Research, vol. 66, no. 10, pp. 5251-5257, 2006.

[64] L. Kleinberg, A. Holth, C. G. Trope, R. Reich, and B. Davidson, "Claudin upregulation in ovarian carcinoma effusions is associated with poor survival," Human Pathology, vol. 39, no. 5, pp. 747-757, 2008.

[65] P. Dhawan, A. B. Singh, N. G. Deane, et al., "Claudin-1 regulates cellular transformation and metastatic behavior in colon cancer," Journal of Clinical Investigation, vol. 115, no. 7, pp. 1765-1776, 2005.

[66] M. B. Resnick, T. Konkin, J. Routhier, E. Sabo, and V. E. Pricolo, "Claudin-1 is a strong prognostic indicator in stage II 
colonic cancer: a tissue microarray study," Modern Pathology, vol. 18, no. 4, pp. 511-518, 2005.

[67] P. D. Leotlela, M. S. Wade, P. H. Duray, et al., "Claudin1 overexpression in melanoma is regulated by $\mathrm{PKC}$ and contributes to melanoma cell motility," Oncogene, vol. 26, no. 26, pp. 3846-3856, 2007.

[68] Y.-L. Wu, S. Zhang, G.-R. Wang, and Y.-P. Chen, "Expression transformation of claudin-1 in the process of gastric adenocarcinoma invasion," World Journal of Gastroenterology, vol. 14, no. 31, pp. 4943-4948, 2008.

[69] H. Miyamori, T. Takino, Y. Kobayashi, et al., "Claudin promotes activation of pro-matrix metalloproteinase-2 mediated by membrane-type matrix metalloproteinases," Journal of Biological Chemistry, vol. 276, no. 30, pp. 28204-28211, 2001.

[70] A. Blanchard, R. Shiu, S. Booth, et al., "Gene expression profiling of early involuting mammary gland reveals novel genes potentially relevant to human breast cancer," Frontiers in Bioscience, vol. 12, pp. 2221-2232, 2007.

[71] A. A. A. Blanchard, P. H. Watson, R. P. C. Shiu, et al., "Differential expression of claudin 1, 3, and 4 during normal mammary gland development in the mouse," DNA and Cell Biology, vol. 25, no. 2, pp. 79-86, 2006.

[72] K. Swisshelm, A. Machl, S. Planitzer, R. Robertson, M. Kubbies, and S. Hosier, "SEMP1, a senescence-associated cDNA isolated from human mammary epithelial cells, is a member of an epithelial membrane protein superfamily," Gene, vol. 226, no. 2, pp. 285-295, 1999.

[73] S. Morohashi, T. Kusumi, F. Sato, et al., "Decreased expression of claudin-1 correlates with recurrence status in breast cancer," International Journal of Molecular Medicine, vol. 20, no. 2, pp. 139-143, 2007.

[74] M. Kulawiec, A. Safina, M. M. Desouki, et al., "Tumorigenic transformation of human breast epithelial cells induced by mitochondrial DNA depletion," Cancer Biology and Therapy, vol. 7, no. 11, pp. 1732-1743, 2008.

[75] T. Hoevel, R. Macek, K. Swisshelm, and M. Kubbies, "Reexpression of the TJ protein CLDN1 induces apoptosis in breast tumor spheroids," International Journal of Cancer, vol. 108, no. 3, pp. 374-383, 2004.

[76] A. A. Blanchard, G. P. Skliris, P. H. Watson, et al., "Claudins 1,3 , and 4 protein expression in ER negative breast cancer correlates with markers of the basal phenotype," Virchows Archiv, vol. 454, no. 6, pp. 647-656, 2009.

[77] Y. Soini, S. Tommola, H. Helin, and P. Martikainen, "Claudins $1,3,4$ and 5 in gastric carcinoma, loss of claudin expression associates with the diffuse subtype," Virchows Archiv, vol. 448, no. 1, pp. 52-58, 2006.

[78] V. Bolos, H. Peinado, M. A. Perez-Moreno, M. F. Fraga, M. Esteller, and A. Cano, "The transcription factor Slug represses E-cadherin expression and induces epithelial to mesenchymal transitions: a comparison with Snail and E47 repressors," Journal of Cell Science, vol. 116, no. 3, pp. 499-511, 2003.

[79] K. M. Hajra, D. Y. Chen, and E. R. Fearon, "The SLUG zincfinger protein represses E-cadherin in breast cancer," Cancer Research, vol. 62, no. 6, pp. 1613-1618, 2002.

[80] O. M. Martinez-Estrada, A. Culleres, F. X. Soriano, et al., "The transcription factors Slug and Snail act as repressors of claudin-1 expression in epithelial cells," Biochemical Journal, vol. 394, no. 2, pp. 449-457, 2006.

[81] J. Kulka, A. M. Szasz, Z. Nemeth, et al., "Expression of tight junction protein Claudin-4 in basal-like breast carcinomas," Pathology and Oncology Research, vol. 15, no. 1, pp. 59-64, 2009.
[82] P. N. Tay, Y. Lan, C. H. Leung, and S. C. Hooi, "Genes associated with metastasis and epithelial-mesenchymal transition (EMT)-like phenotype in human colon cancer cells: palladin in colon cancer metastasis," in Proceedings of the 99th Annual Meeting of the American Association for Cancer Research (AACR '08), San Diego, Calif, USA, April 2008, abstract no. 5361.

[83] J. I. Herschkowitz, K. Simin, V. J. Weigman, et al., "Identification of conserved gene expression features between murine mammary carcinoma models and human breast tumors," Genome Biology, vol. 8, article R76, 2007. 

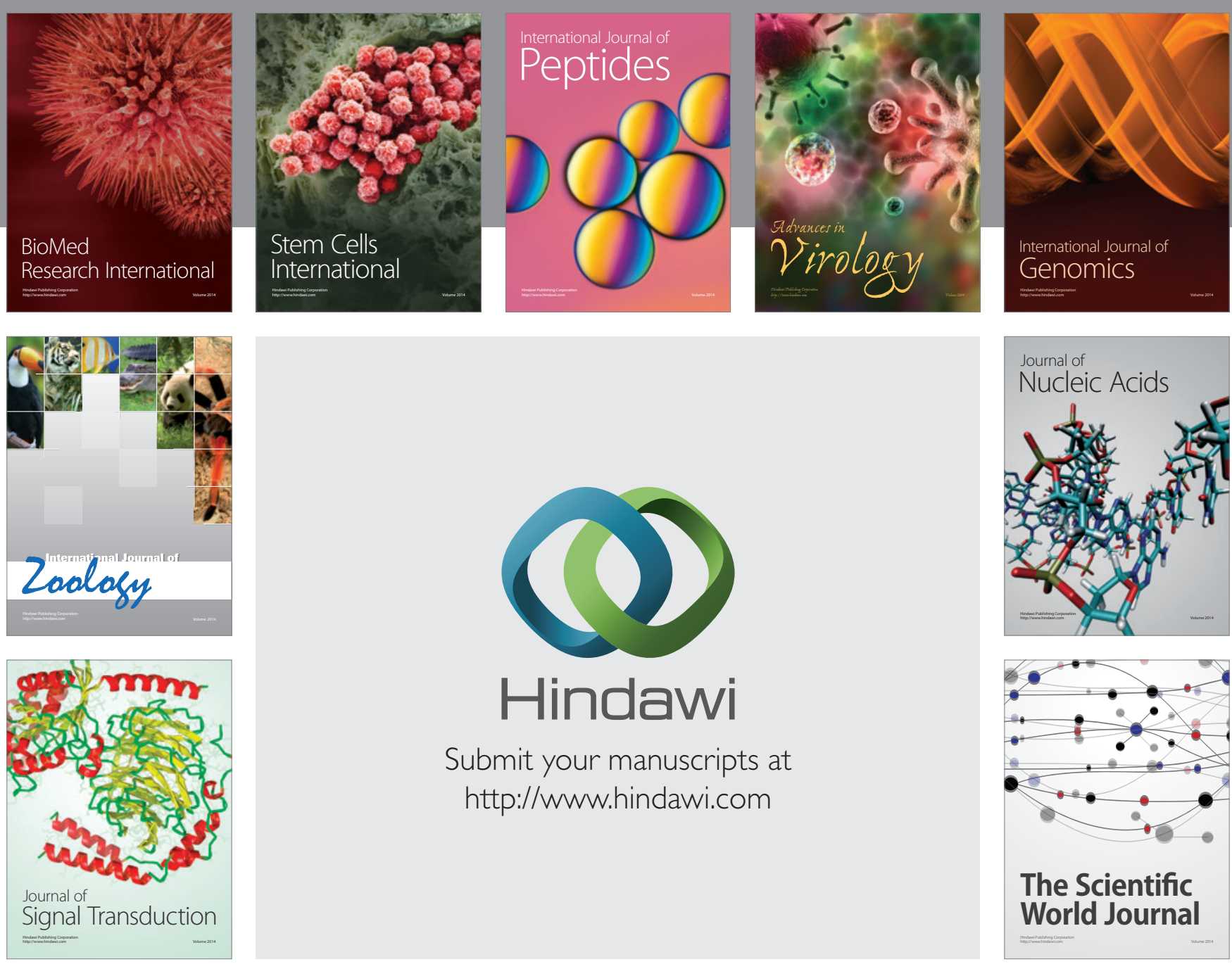

Submit your manuscripts at

http://www.hindawi.com
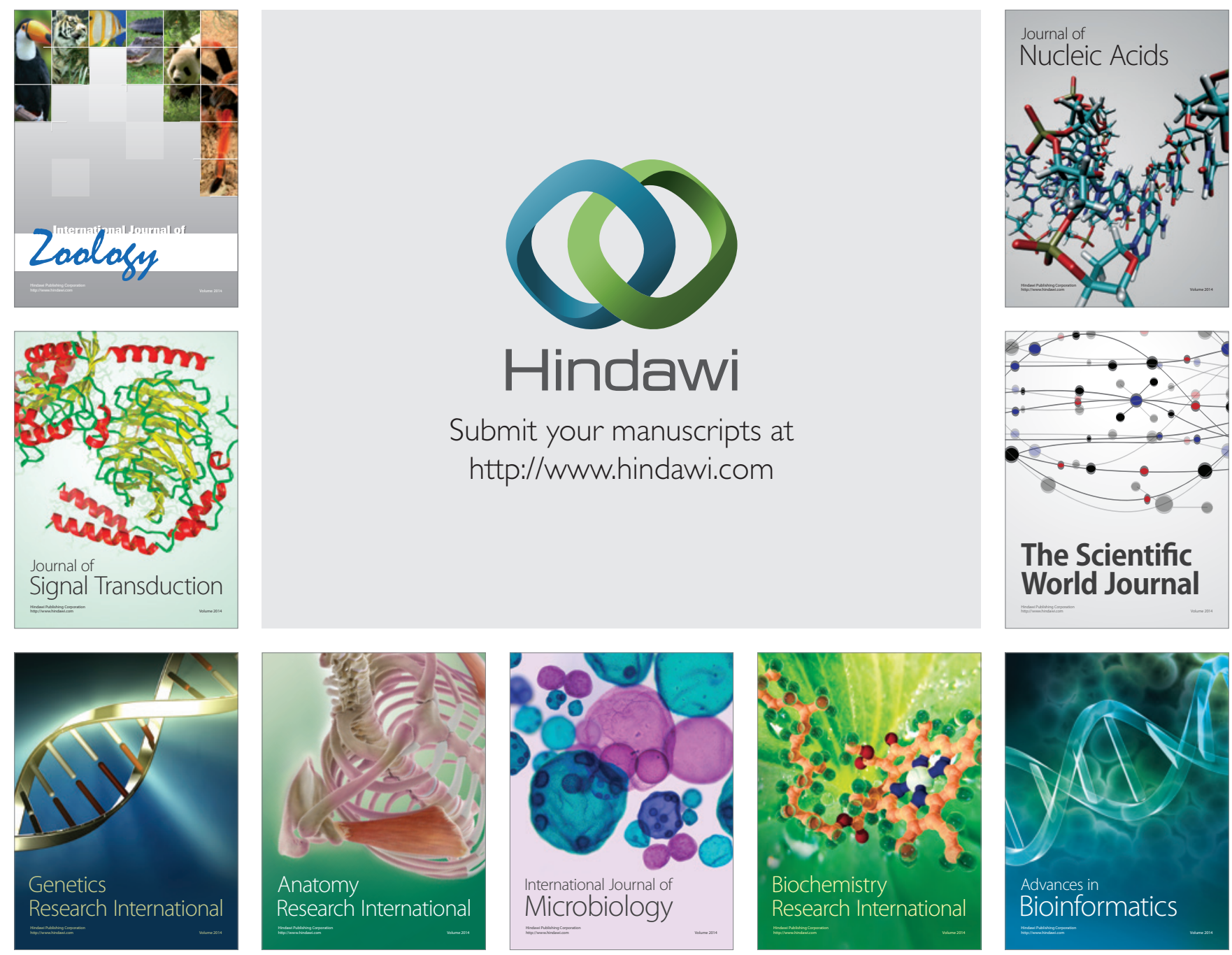

The Scientific World Journal
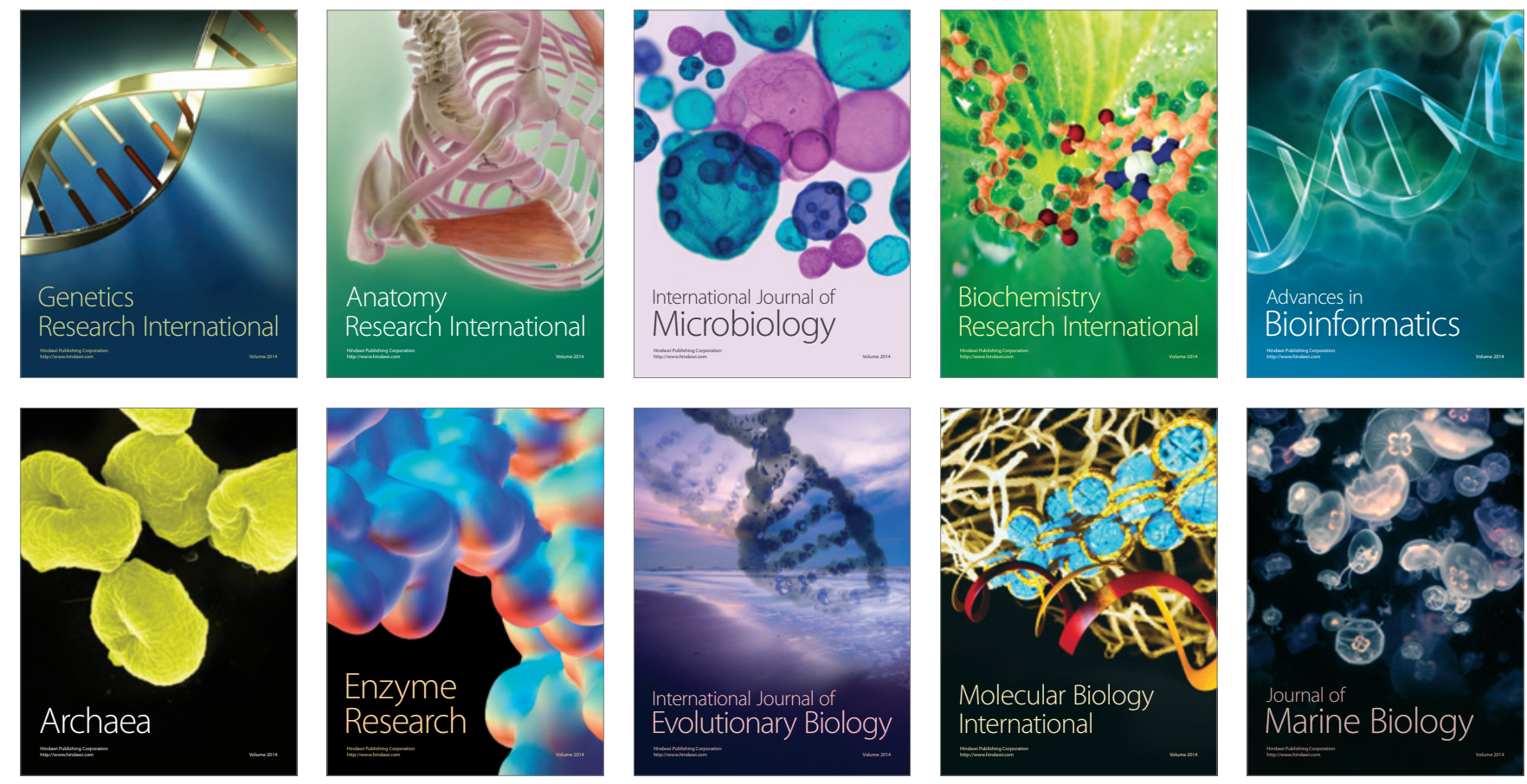